\title{
QWIPs, SLS, Landsat and the International Space Station
}

\section{Murzy Jhabvala, Kwong-Kit Choi, Sarath Gunapala, Manijeh Razeghi, Mani Sundaram}

Murzy Jhabvala, Kwong-Kit Choi, Sarath Gunapala, Manijeh Razeghi, Mani Sundaram, "QWIPs, SLS, Landsat and the International Space Station," Proc. SPIE 11288, Quantum Sensing and Nano Electronics and Photonics XVII, 1128802 (31 January 2020); doi: 10.1117/12.2539147

SPIE. Event: SPIE OPTO, 2020, San Francisco, California, United States 


\title{
QWIPs, SLS, Landsat and the International Space Station
}

\author{
Murzy Jhabvala ${ }^{1}$, Kwong-Kit Choi $^{2}$, Sarath Gunapala ${ }^{3}$, Manijeh Razeghi ${ }^{4}$, Mani Sundaram ${ }^{5}$ \\ ${ }^{1}$ NASA Goddard Space Flight Center, Greenbelt, Maryland 20771 USA \\ ${ }^{2}$ Science Systems and Applications, Inc., Lanham, Maryland 20706 \\ ${ }^{3}$ Jet Propulsion Laboratory, 4800 Oak Grove Drive, Pasadena, California 91109, USA \\ ${ }^{4}$ Center for Quantum Devices, Northwestern University, Evanston, Illinois 60208, USA \\ ${ }^{5}$ QmagiQ, LLC, 22 Cotton Road, Unit H, Suite 180, Nashua, NH 03063 USA
}

\begin{abstract}
In 1988 DARPA provided funding to NASA's Goddard Space Flight Center to support the development of GaAs Quantum Well Infrared Photodetectors (QWIP). The goal was to make a single element photodetector that might be expandable to a two-dimensional array format. Ultimately, this led to the development of a $128 \times 128$ element array in collaboration with AT\&T Bell Labs and Rockwell Science Center in 1990. We continued to develop numerous generations of QWIP arrays most recently resulting in the multi-QWIP focal plane for the NASA-US Geological Survey (USGS) Landsat 8 mission launched in 2013 and a similar instrument on the Landsat 9 mission to be launched in 2020. Toward the end of the Landsat 8 QWIP-based Thermal Infrared Sensor (TIRS) instrument the potential of the newly developed Strained Layer Superlattice (SLS) detector array technology became of great interest to NASA for three primary reasons: 1) higher operating temperature; 2) broad spectral response and; 3) higher sensitivity. We have collaborated extensively with QmagiQ, LLC and Northwestern University to further pursue and advance the SLS technology ever since we started back in 2012. In December of 2018 we launched the first SLS-based IR camera system to the International Space Station on board the Robotic Refueling Mission \#3 (RRM3). This paper will describe the evolution of QWIP technology leading to the current development of SLS-based imaging systems at the Goddard Space Flight Center over the past 30 years.
\end{abstract}

\section{INTRODUCTION}

The evolution of both GaAs QWIP and SLS technologies has been remarkable [1]. In 1989, we fabricated a single element QWIP detector that had an angled edge for optical coupling. As primitive as this device was it gave valuable credence to internal research and development proposals requesting minimal funding to further pursue and advance this technology particularly for NASA earth observing missions. Subsequent to this initial single element QWIP detector we developed:

- 128 x 128 QWIP array hybrid with AT\&T and Rockwell Science Center

- 256 x 256 E-QWIP array hybrid with LTV

- 640 x 512 4-band/hyperspectral QWIP array hybrid with ARL and JPL

- $\quad 640$ x 512 8-13 $\mu \mathrm{m}$ QWIP array hybrid with ARL, QmagiQ and Thales

- $\quad 1 \mathrm{k} x$ 1k QWIP 8-13 $\mu \mathrm{m}$ array hybrid with ARL, JPL and Rockwell Science Center

- Voltage selectable MWIR/LWIR 256 x 256 QWIP array hybrid with Raytheon Vision Systems

- $\quad 2 \mathrm{k} x$ 2k QWIP array with ARL and Raytheon

- $\quad 512$ x 3k QWIP array

Of particular importance was the development of the $640 \times 512,8-13 \mu \mathrm{m}$ spectral response QWIP array hybridized to an Indigo ISC9803 readout integrated circuit (ROIC). It was this detector that was selected for the Landsat 8 focal plane that was launched into orbit February $11^{\text {th }}, 2013$. Subsequently, it was decided that this exact focal plane would also be used on the Landsat 9 mission that is expected to be launched in 2020.

Quantum Sensing and Nano Electronics and Photonics XVII, edited by Manijeh Razeghi,

Jay S. Lewis, Giti A. Khodaparast, Pedram Khalili, Proc. of SPIE Vol. 11288, 1128802

(C) 2020 SPIE · CCC code: $0277-786 X / 20 / \$ 21 \cdot$ doi: $10.1117 / 12.2539147$ 


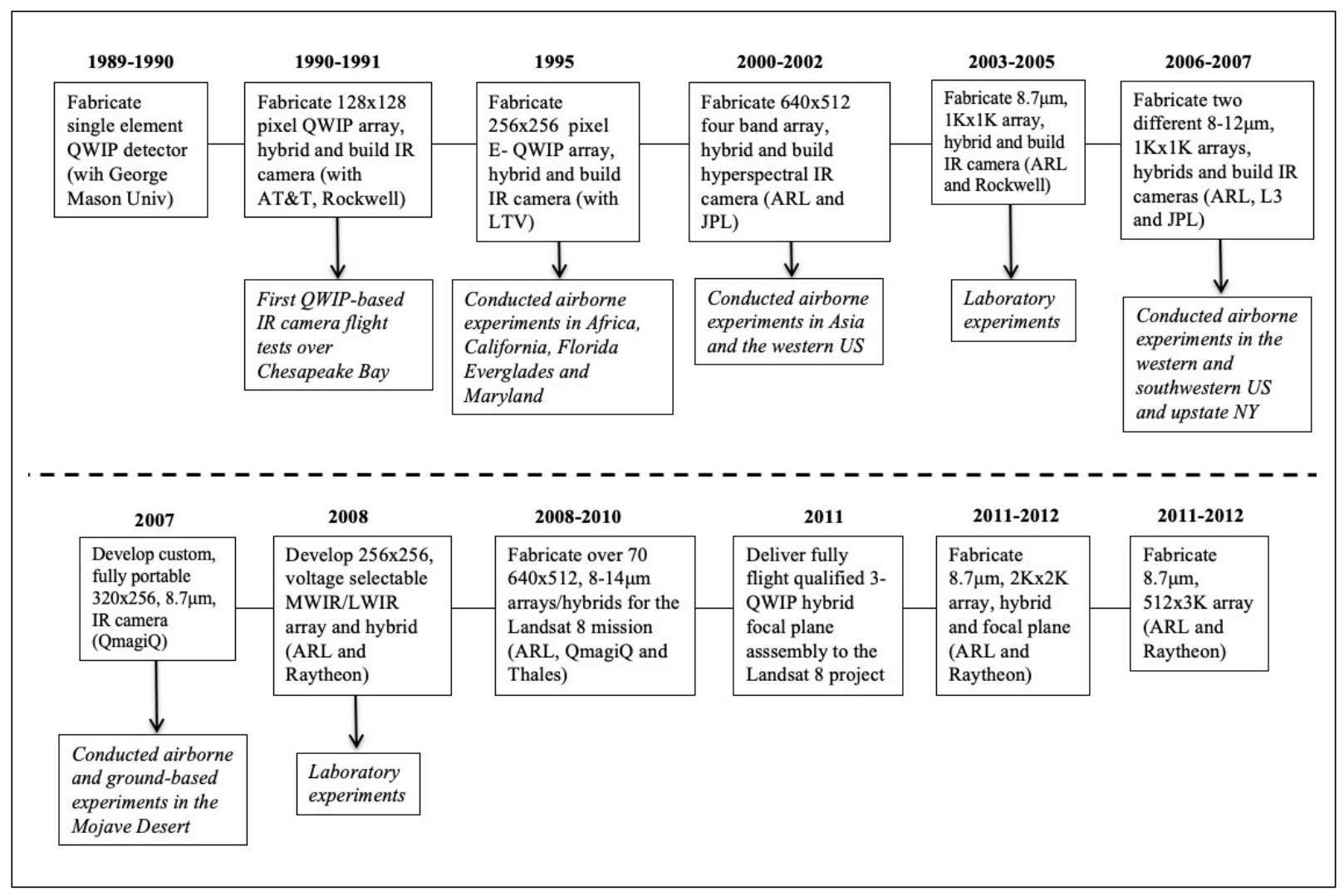

Figure 1. History of the development of QWIP detector arrays at the Goddard Space Flight Center (and collaborators) spanning more than two decades.

The above block diagram illustrates the evolution of the QWIP development spanning three decades at the NASA/Goddard Space Flight Center. Every program was a collaborative effort, but the most enduring collaboration was the partnership with the US Army Research Laboratory. Subsequently, collaborations with JPL, QmagiQ and NOUR, Inc developed and have also endured and have been key in our development of devices of interest to NASA engineers and scientists.

Once the focal plane development for the Landsat 8 satellite was completed in 2011, we started exploring the relatively new and emerging strained-layer superlattice technology. A number of factors were evident from the Landsat project. The QWIP arrays needed to be cooled to $42 \mathrm{~K}$, the sensitivity was quite low, and the spectral response was difficult to broaden. The SLS technology appeared to surmount these hurdles. The spectral response could cover the $4-13 \mu \mathrm{m}$ wavelength region while the operating temperature could be increased to near $77 \mathrm{~K}$. The quantum efficiency across the spectrum could average well above $20 \%$, an order of magnitude improvement over existing QWIPs. Almost too good to be true. To overcome our initial skepticism about the performance of SLS detectors we collaborated with QmagiQ through the Goddard Internal R\&D and NASA SBIR programs to develop a 640 × 512 SLS detector hybrid using a FLIR/Indigo 0402 ROIC. The format was identical to the QWIP array format on Landsat 8, but the pixel size was $20 \times 20 \mu \mathrm{m}$ vs 25 $\mu \mathrm{m}$ for the QWIP hybrids on Landsat 8. A side by side comparison between the two technologies confirmed the claims about the SLS performance. From this point on we focus our attention on developing and exploiting the SLS technology. The block diagram in figure 2 illustrates the path and progress of SLS technology development in collaboration with Goddard and our various partners. Compared to the 20 years from the initial development of QWIP detectors at NASA to implementation into a successful NASA space-flight mission, the SLS technology achieved a somewhat similar milestone in a little more than 5 years (clearly from a NASA perspective). The general SLS development at Goddard is listed below. Again, the efforts were extremely collaborative primarily with QmagiQ and Northwestern University and NOUR. 
- 2-band electrically switchable 320 x 256 SLS Integrated Detector Cooler Assembly (IDCA) with QmagiQ and Northwestern/NOUR

- $\quad 640 \times 512$ broadband (4-12 $\mu \mathrm{m})$ SLS hybrid with QmagiQ

- 640 x 512 SLS NIR camera with QmagiQ

- $\quad 1 \mathrm{k}$ x 1k SLS hybrids and camera with QmagiQ and Northwestern/NOUR

- 2-band electrically switchable 1280 x 1024 MW/FIR SLS hybrids and camera with QmagiQ and NOUR

- 640 x 512 SLS Vis-NIR camera with QmagiQ

- $\quad 2 \mathrm{k} \times 2 \mathrm{k}$ SLS hybrid with QmagiQ

- 640 x 512 SLS hybrid with direct attached 5-band butcher block filter at Goddard

- $\quad 1 \mathrm{k}$ x 1k SLS hybrid with direct attached 5-band butcher block filter with QmagiQ

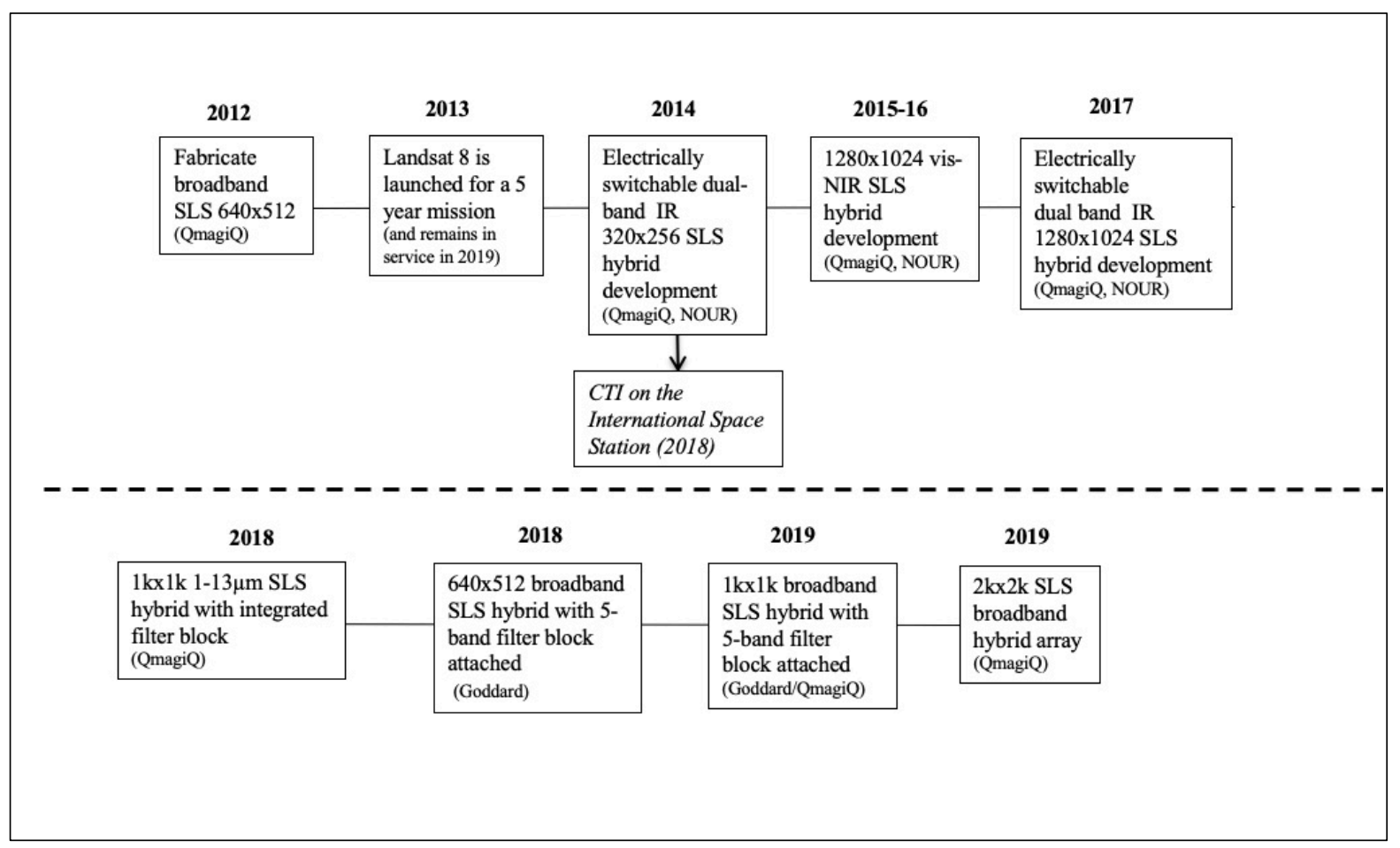

Figure 2. Recent development of SLS detector arrays at the Goddard Space Flight Center (and collaborators).

\section{Quantum Well Photodetectors for the Landsat 8 and Landsat 9 Missions}

The development of GaAs QWIPs at Goddard began with the fabrication and characterization of a single element detector. In hindsight this may seem like a rudimentary goal but the Cosmic Background Explorer satellite that was launched in 1989 to confirm the "big bang theory" contained only single element detectors. Infrared arrays were just beginning to emerge. The first $128 \times 128$ [2] QWIP array we developed was a collaborative effort between AT\&T bell Labs, Rockwell Science Center and Goddard in 1990-91The next generation of QWIP arrays was based on an LTV developed 256 $\times 256$ enhanced or E-QWIP design. We conducted numerous field experiments which captured the interest of the earth science 
community at Goddard. Our next development was a collaboration with engineers at JPL to develop a hyperspectral QWIP array spanning the IR spectrum, in four discreet bands, from 3-15.4 $\mu \mathrm{m}$. The array was a $640 \times 512$ and the four bands, each $640 \times 128$, were 1) 3-5 $\mu \mathrm{m}$; 2) $8.5-10 \mu \mathrm{m}$; 3) $10-12 \mu \mathrm{m}$ and; 4) $14-15.4 \mu \mathrm{m}$. The bands were further sub-divided by a linear variable etalon filter such that the $3-5 \mu \mathrm{m}$ band was subdivided into 100 discreet bands; the $8.5-10 \mu \mathrm{m}$ band was subdivided into 37 discreet bands; the 10-12 $\mu \mathrm{m}$ band was subdivided into 44 discreet bands and; the 14-15.4 $\mu \mathrm{m}$ band was subdivided into 28 discreet bands. This was the first time that a QWIP imaging array provided both spatial and hyperspectral scene information [3]. Based upon the success of this collaborative effort, a subsequent collaboration starting in 2002 was undertaken to develop a $1 \mathrm{~K} \times 1 \mathrm{~K}$ broadband 8-14 $\mu \mathrm{m}$ far IR array. As a result of this developmental effort we were able to demonstrate a number of technology solutions that ultimately led to consideration of this technology for a major NASA space-flight mission. Concurrent with this program we pursued the implementation of a mechanical cryocooler which was a major improvement in performing IR experiments. Subsequently, QmagiQ, LLC of Nashua, NH was contracted to build a completely integrated IR imaging system in a portable video camera configuration [4]. This system has been used for over 10 years in numerous ground-based and airborne experiments.

The successful QWIP technology development resulted in the selection of 640x512 format QWIP arrays being selected as the focal plane technology for the Earth-observing thermal infrared imaging instrument build for the Landsat 8 mission that was launched in 2013. This QWIP development program was a collaboration between Goddard, ARL, QmagiQ, LLC and Thales Research and Technology (Palaiseau, France) [5]. Images of the Landsat 8 and 9 TIRS focal plane is shown below in figure 3.

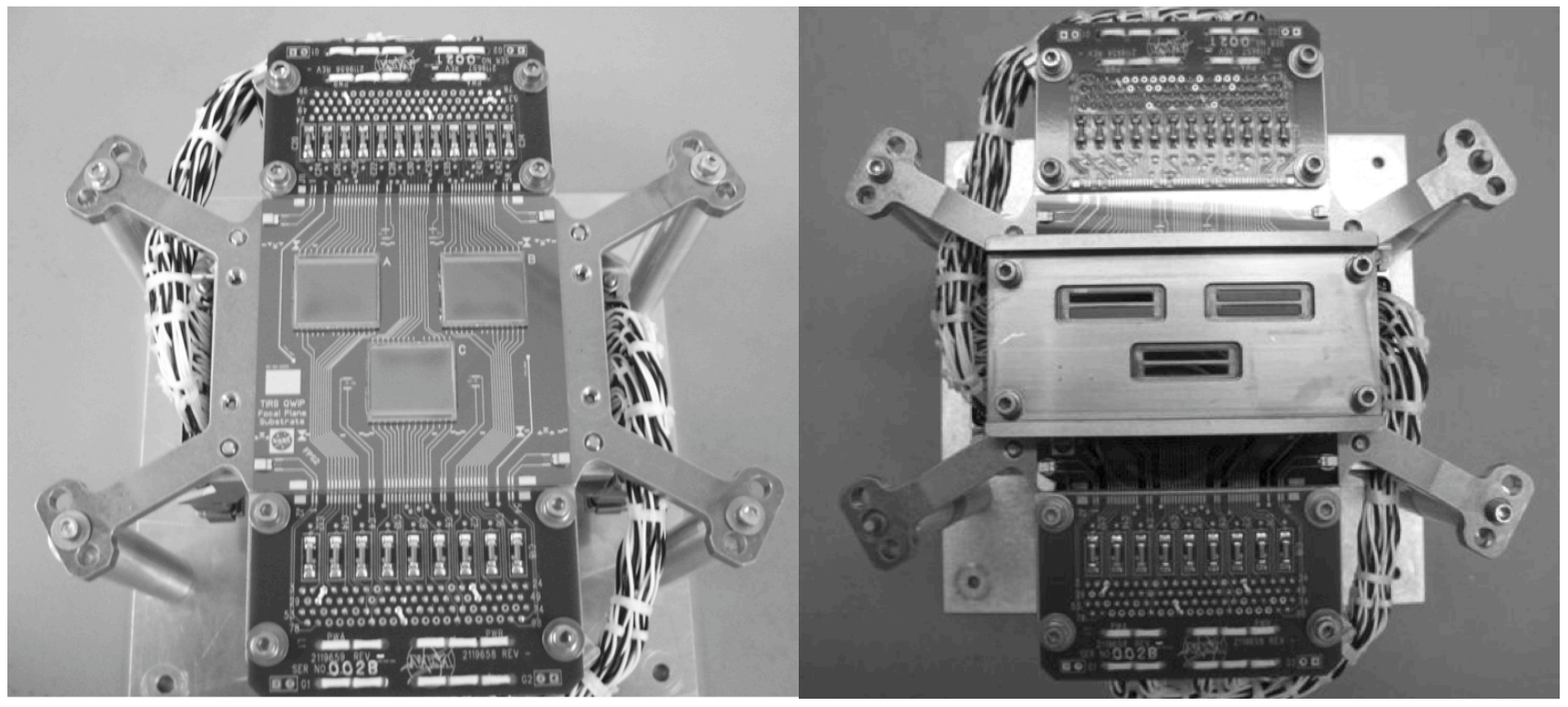

Figure 3. The Landsat 8 and 9 Thermal Infrared Imager focal planes without (left) and with (right) the band selection filters.

In 18 months over seventy $640 \times 512$ QWIP hybrids were fabricated, tested and qualified. Each hybrid was thoroughly evaluated; signal versus integration time (linearity), dark current vs. detector bias voltage and temperature, relative spectral response, absolute conversion efficiency versus wavelength, 45-minute dark current stability, 45-minute absolute CE stability, noise performance, $\mathrm{NE} \Delta \mathrm{T}$ compliance, non-functional pixels, full well capacity, power dissipation and spectral uniformity. The culmination of 20+ years of QWIP R\&D led to a successful mission with the detector performance shown in Table 1. Subsequently, the identical format QWIP-based focal plane was implemented in the Landsat 9 mission to be launched in 2020. Shown below in figure 4 are TIRS images from Landsat 8 of the Larsen C Ice Shelf before and after the rift occurred creating the A-68 iceberg in 2017. 
From Table 1 it is clear that there two major drawbacks; operating temperature and low sensitivity (CE). Since the Landsat mission is Earth observing the low sensitivity is not an issue - there is plenty of signal emanating from the Earth. However, the $43 \mathrm{~K}$ operating temperature is problematic requiring special cooling considerations. Consequently, the development of the SLS technology rapidly overtook the QWIP technology; SLS detectors operate at warmer temperatures, have a higher sensitivity and the spectral response can be much broader. However, in spite of these current drawbacks the performance of the QWIP detectors on Landsat has been quite remarkable and revealing.

\section{A. QWIP Stability}

Approximately 2 years after the Landsat 8 was launched an anomaly in the on-board encoder occurred which prevented the internal TIRS blackbody calibration sources from being accessed. As a result, the normal twice-an-orbit calibration (every 90 minutes) was not possible. An alternative calibration scenario allowed for calibration once every two weeks and no degradation in instrument performance was observed [6]. Ground based QWIP imaging systems that have been installed at astronomical observatories in Arizona (Kitt Peak) and California (Big Bear) have confirmed this stability over a 5-year period. This intrinsic stability of the III-V material system is a very attractive feature in scientific instrumentation where size, power and weight are at a premium. The elimination

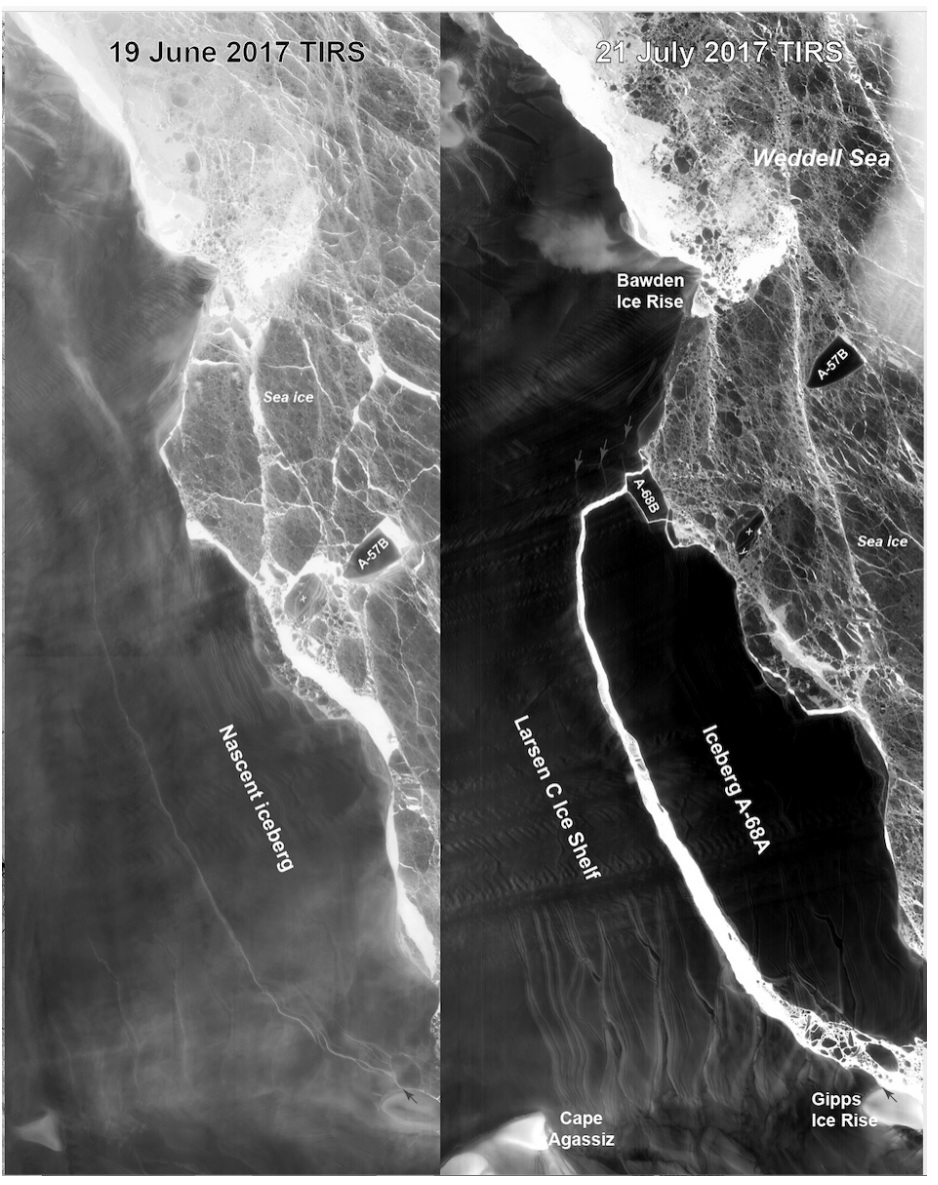

Figure 4. Example of a Landsat TIRS image. Two images of the Larsen C Ice Shelf in the Antarctica approximately one month apart in 2017. The images show the formation of the Iceberg A-68A. This rift reduced the ice shelf by 2,200 square miles (but does not affect the global sea level).

of an on-board calibration instrument is a significant reduction in mission complexity. This has been a big selling feature in Earth-observing remote sensing instruments and has led to the proliferation of numerous NASA instrument concepts. Combined with a higher quantum efficiency, this class of detector is quite attractive and hence the concerted drive to develop and implement SLS detectors into these instruments. 
Table 1. Performance of Landsat 8 and 9 QWIP arrays

\begin{tabular}{|l|l|}
\hline Detector operating temperature & $43 \mathrm{~K}$ \\
\hline Array format & $640 \mathrm{x} 512$ \\
\hline Pixel pitch & $25 \mu \mathrm{mx} 25 \mu \mathrm{m}$ \\
\hline FWHM spectral response & $9.5-12.8 \mu \mathrm{m}$ \\
\hline Dark current & $8 \times 10^{7} \mathrm{e} / \mathrm{sec} / \mathrm{pixel}$ \\
\hline Peak wavelength response & $11.1 \mu \mathrm{m}$ \\
\hline Average conversion efficiency $(\mathrm{CE})$ at $\lambda=11.1 \mu \mathrm{m}$ & $2.3 \%$ \\
\hline Average NE $\Delta \mathrm{T}$ & $16.1 \mathrm{mK}$ \\
\hline Pixel-pixel spectral non-uniformity & $<3 \%$ \\
\hline CE stability & $<0.4 \%$ over 45 minutes \\
\hline Dark current variation & $<0.2 \%$ over 45 minutes \\
\hline
\end{tabular}

\section{Strained Layer Superlattice Array Development}

The development of SLS technology has been much more rapid than that of QWIP technology primarily due to all the technological achievements that were realized during the evolution of QWIPs. The development and refinement of MOCVD and MBE processing, the advent of mechanical coolers and the availability of a wide variety of ROICs at relatively affordable costs all contributed to the very rapid development of sophisticated SLS arrays. The acceptance of QWIP technology throughout the scientific community was slow and hard to implement. However, after achieving technical credibility with QWIP technology (via Landsat 8) gaining the acceptance of SLS technology was a much easier road to plow. At Goddard this SLS progress was enabled with the collaboration of two organizations; QmagiQ, LLC and Northwestern University (with NOUR Inc,). Goddard and QmagiQ and Goddard and Northwestern University embarked on over a dozen different SLS-based projects in the last decade starting with the development of a broadband, 640x512 SLS array on the ISC9803 ROIC. The spectral response spanned 3-12 $\mu \mathrm{m}$ with an average QE $>20 \%$. It was this device that convinced many of us that the test results being reported in the literature [7] were readily achievable with existing technology and fabrication capabilities [8]. In short, it was a very easy processing leap to take with an almost instantaneous improvement (by more than an order of magnitude) in sensitivity, range of spectral selectivity and normal incidence to the optical path-from near, for far and more generally, broadband IR imaging - all deficiencies experienced 

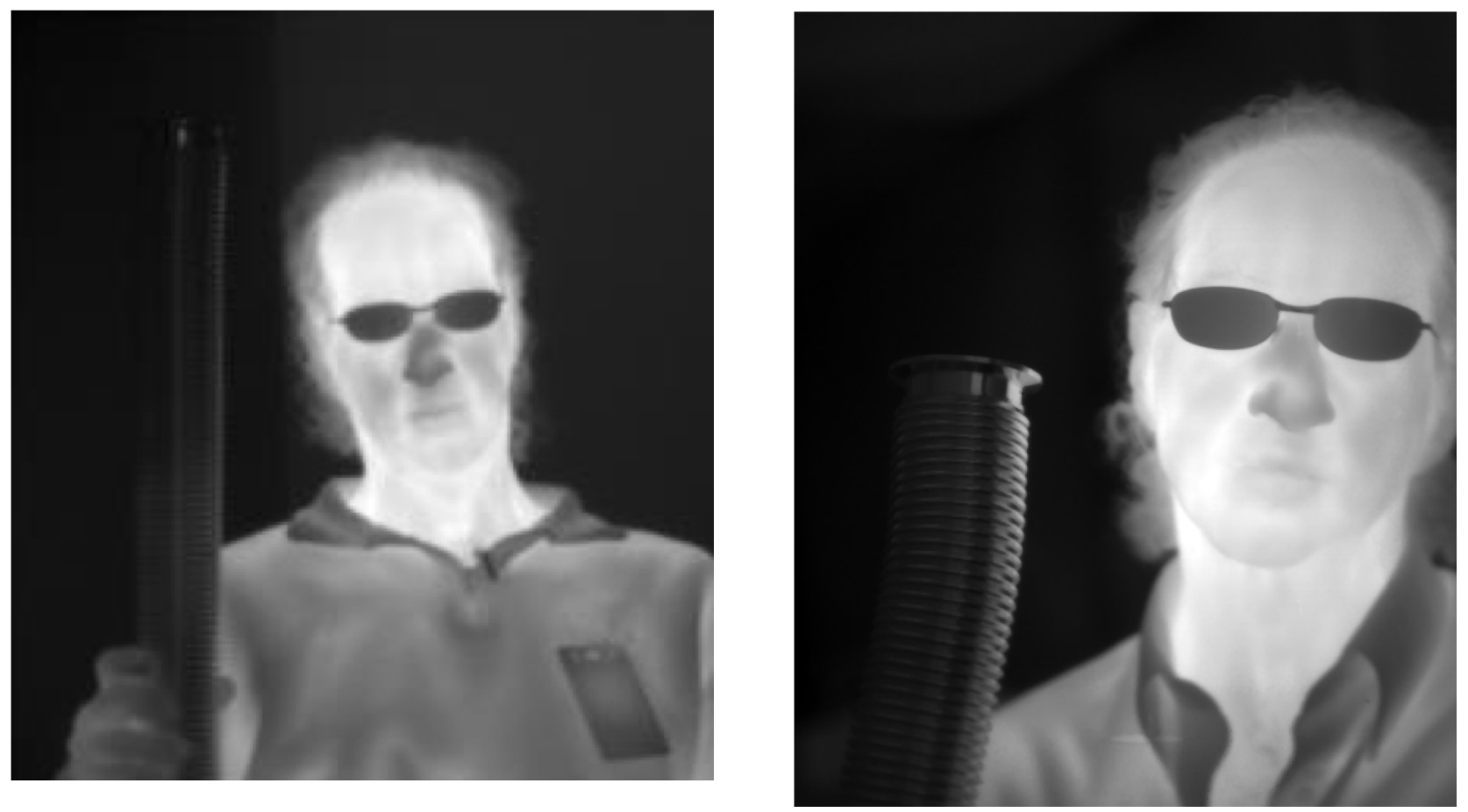

Figure 5. Left; QWIP narrow band image. Right; First SLS broadband IR image taken in the Goddard lab. Subjectively, the images looked comparable but the integration time for the SLS image was almost 90 times shorter than for the QWIP image due to the increased sensitivity (0.19 ms vs $16.65 \mathrm{~ms})$.

in the earlier QWIP technology. Shown below in figure 5 are images taken with a laboratory narrow band $8.5 \mu \mathrm{m}$ QWIP camera and with the broadband 640x512 SLS-based camera. Aside from the comparable image quality the most remarkable test result was that the SLS camera could operate near $80 \mathrm{~K}$ (with $\lambda_{c}>12 \mu \mathrm{m}$ ). Given that the Landsat QWIP-based TIRS instruments needed to operate at $42 \mathrm{~K}$ (for $\lambda>10.5 \mu \mathrm{m}$ ) this was a remarkable achievement. The level of complexity to cool from $80 \mathrm{~K}$ to below $\sim 50 \mathrm{~K}$ is enormous for satellite missions. Aside from the improvement in all the other SLS performance parameters this increase in operating temperature is likely the single most valuable improvement for NASA missions (particularly Earth-viewing and planetary missions). Subsequent R\&D programs led to the development of large format arrays (1280x1024) that could be electrically switched between two spectral bands by reversing the detector bias in alternating image frames. A 320x256 precursor version of this electrically switchable dual band detector was ultimately re-packaged into an imager that was successfully launched to the International Space Station as the Compact Thermal Imager discussed in the next section. Another important development by QmagiQ and Goddard was the development of a fully autonomous $1 \mathrm{kx} 1 \mathrm{k}, 4-13 \mu \mathrm{m}$ camera system which was used to perform airborne imaging over US Department of Agriculture experimental crop fields in Beltsville, Maryland which also happens to be adjacent to the Goddard Space Flight Center. Both continuous video and 16-bit images (collected every 2 seconds) were acquired. The camera was installed in the wing pod of a Cessna $206 \mathrm{H}$ aircraft flying at 2,000 and 4,000 feet. The image ground resolution was approximately $0.8 \mathrm{ft} / \mathrm{pixel}$ at the $2,000 \mathrm{ft}$ altitude. Shown below in figure 6 is one frame of the flight sequence consisting of approximately 2,000 images. One of the challenges of airborne instruments is making it operable by the aircraft crew. Often, having the experimenter fly along with the instrument is not an option so the pilot or co-pilot needs to be trained in operating the instrument which can be simple or complicated. We had configured this camera so that we could either 1) turn it on before take-off which will give us a lot of useless data or 2) have the pilot just push a 


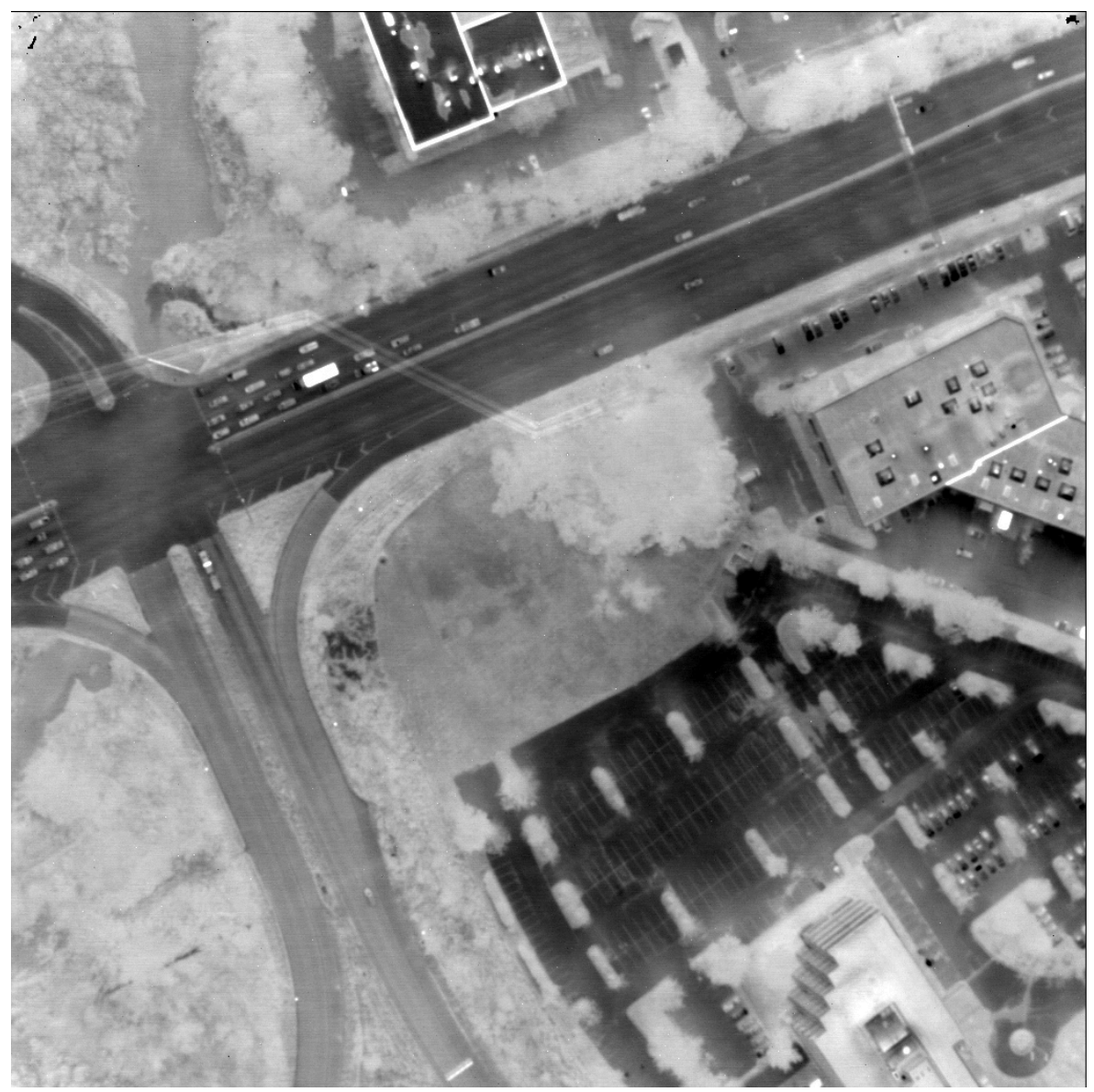

Figure 6. Ariel image taken with the $1 \mathrm{kx} 1 \mathrm{k}$ SLS camera from an altitude of 2,000 feet.

start/stop switch at the appropriate times. In either case the camera would run unattended which was an important demonstration for applications where the instrument must operate autonomously. Subsequent to the airborne experiments we contacted the BaltimoreWashington airport authority to allow us to bring this camera to the airport to observe airfield activities. Shown below in figure 7 is an IR image of the BWI airfield taken with the $1 \mathrm{kx} 1 \mathrm{k}$ autonomous imaging system. The thermal contrast between the jet engines and warm tires is quite striking particularly the exhaust gas from the engines. The images were taken with a $100 \mathrm{~mm}$ IR lens. Concurrent with the SLS technology progress was also the development of miniature mechanical coolers. Together these developments have spawned a number of NASA mission concepts that could now fit in a CubeSat configuration [9]. A single

CubeSat envelope is $10 \mathrm{~cm} \times 10 \mathrm{~cm} \times 10 \mathrm{~cm}$ (1U CubeSat). Instruments are designed for 3, 6 and 12 times a $1 \mathrm{U}$ CubeSat volume. The combination of warmer operating temperature and the advent of miniature cryocoolers has spawned a myriad of instrument concepts in the CubeSat format. By launching a single CubeSat to literally dozens at a time, enormous scientific capabilities can be easily and inexpensively achieved in a relatively short time frame. 


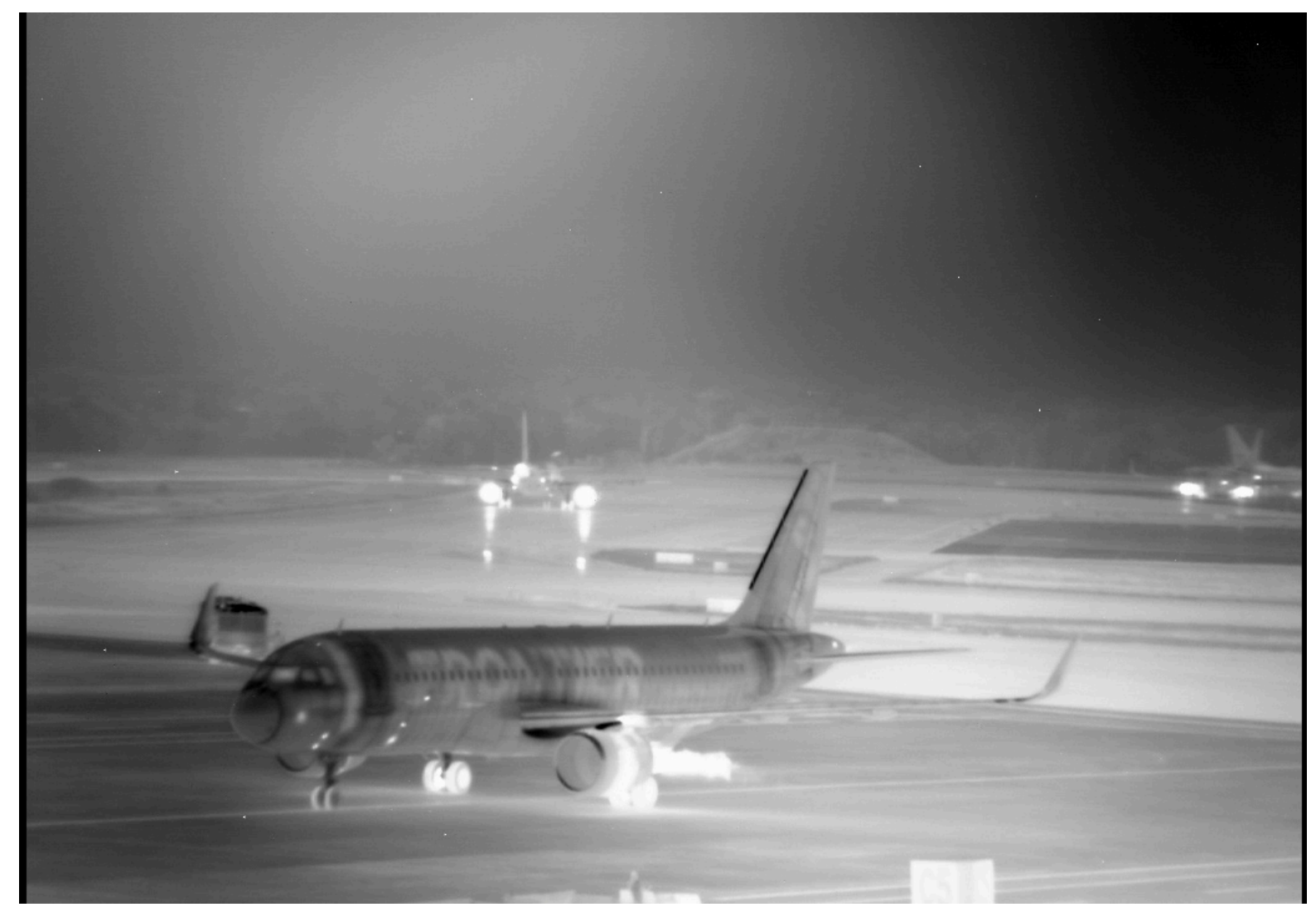

Figure 7. IR image of the Baltimore-Washington International airport airfield activities.

We were able to convert one of our experimental IDCA SLS-based units we received from an SBIR with QmagiQ and convert it into a space-qualified imaging system that was launched to and installed on the International Space Station. This instrument is described in the next section.

\section{The Compact Thermal Imager (CTI)}

An opportunity arose in late 2015 to perform a technology validation of an SLS-based instrument in conjunction with the third Robotic Refueling Mission on the International Space Station (ISS). An SBIR collaboration with QmagiQ [10] led to the development of 320x256 SLS array installed in a Ricor K508 cryocooler. This SLS detector is an electrically switchable dual-band array that was hybridized to an ISC0903 ROIC. There is substantial overlap between the two bands. A warm blocking filter was added to further distinguish the two bands. The resulting spectral response is shown in figure 8 along with the QmagiQ pixel design. 

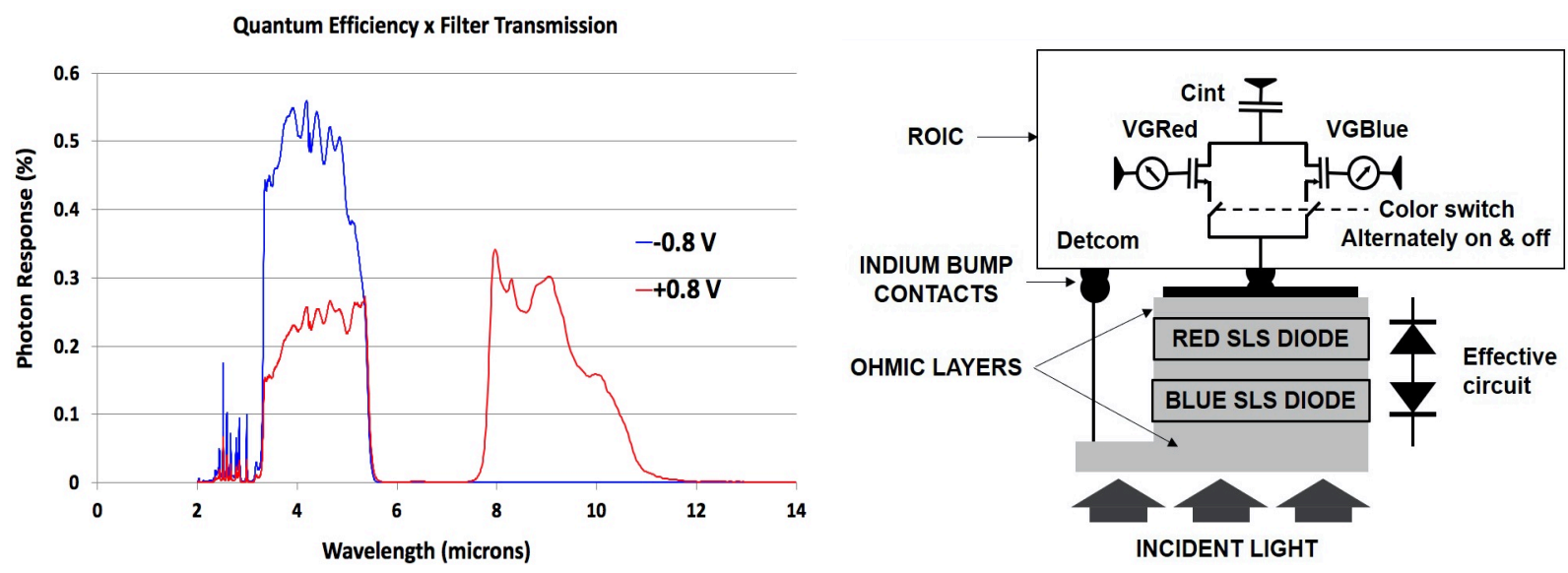

Figure 8. Left: The two spectral responses including the filter transmission when the detector bias is toggled between +0.8 and -0.8 volts. Right: Schematic of the dual band pixel-ROIC layout. The two SLS diodes are grown one on top of the other with the longer response band above the shorter response band.

The focal plane characteristics are listed below in Table 2.

Table 2. Characteristics of the Focal Plane

\begin{tabular}{|l|l|}
\hline \multicolumn{1}{|c|}{ Parameter } & \\
\hline Array format & $320 \times 256$ \\
\hline Pixel pitch & $30 \mu \mathrm{m}$ \\
\hline Detector operating temperature & $\sim 81 \mathrm{~K}$ \\
\hline ROIC & ISC0903 \\
\hline Band 1-MWIR band response (without filter installed); & $2.2-5.4 \mu \mathrm{m}$; fire channel \\
integration time: & $1.8 \mathrm{~ms}$ \\
\hline Band 2-LWIR band response (without filter installed); & $2.2-10.5 \mu \mathrm{m}$ thermal channel \\
integration time: & $8 \mathrm{~ms}$ \\
\hline Band 2-LWIR band response (with filter installed) & $7.8-10.5 \mu \mathrm{m}$ \\
\hline Focal length & $150 \mathrm{~mm}$ \\
\hline f-number & 3.3 \\
\hline Band 1 average quantum efficiency & $>50 \%$ \\
\hline Band 2 average quantum efficiency & $>30 \%$ \\
\hline Band 1 NE $\Delta \mathrm{T}$ & $\sim 16 \mathrm{mK}$ \\
\hline Band 2 NE $\Delta \mathrm{T}$ & $\sim 27 \mathrm{mK}$ \\
\hline Altitude & $\sim 400 \mathrm{~km}$ \\
\hline Ground resolution & $\sim 80 \mathrm{~m} /$ pixel \\
\hline Swath width & $20.5 \times 25.6 \mathrm{~km}$ \\
\hline Pointing knowledge $<5 \mathrm{~km}$ & Better than $5 \mathrm{~km}$ using ISS supplied \\
\hline
\end{tabular}


A photograph of the CTI is shown below in figure 9. The CTI was successfully launched to the ISS aboard the RRM3 experiment on December $5^{\text {th }}, 2018$ on the SpaceX $16^{\text {th }}$ Commercial Resupply Services (CRS-16) mission [11]. The first images were received February $15^{\text {th }}, 2019$ as the ISS crossed over western Africa. The initial goals of CTI were technology demonstrations:

- To demonstrate and validate SLS detector technology in a space environment

- To demonstrate and validate SLS/ROIC hybridization technology

- To demonstrate and validate the Ricor K508 Stirling cooler (this cooler had been flown previously on the Messenger mission [12]

- To utilize commercially available optical components

- To demonstrate and validate methods of adhesion (not to be trivialized)

- To demonstrate and validate the autonomous/remote control by SpaceCube[13]

All these goals were achieved very soon after the CTI was deployed in February. Since that time CTI has been taking images over the Earth to assess its scientific capabilities. To this end we have acquired over 10 million images in a 5month period and continue to collect images. Compared to this number a very small subset has been analyzed. CTI is operated in a continuous snapshot mode. Every two seconds a full-frame image is captured in each of the two bands or one image/second. This frequency provides for continuous Earth coverage with an overlap of about $20 \%$ between subsequent images in the same band. CTI is fixed on the ISS and thus images a swath dictated by the ISS orbit. As the fires raged in the Amazon this past summer numerous images were captured. One such image is shown in Figure 10. The CTI image in band 1 is superimposed on a Google Earth image of the same area. This image was taken on August 1, 2019 as the ISS crossed over South America.
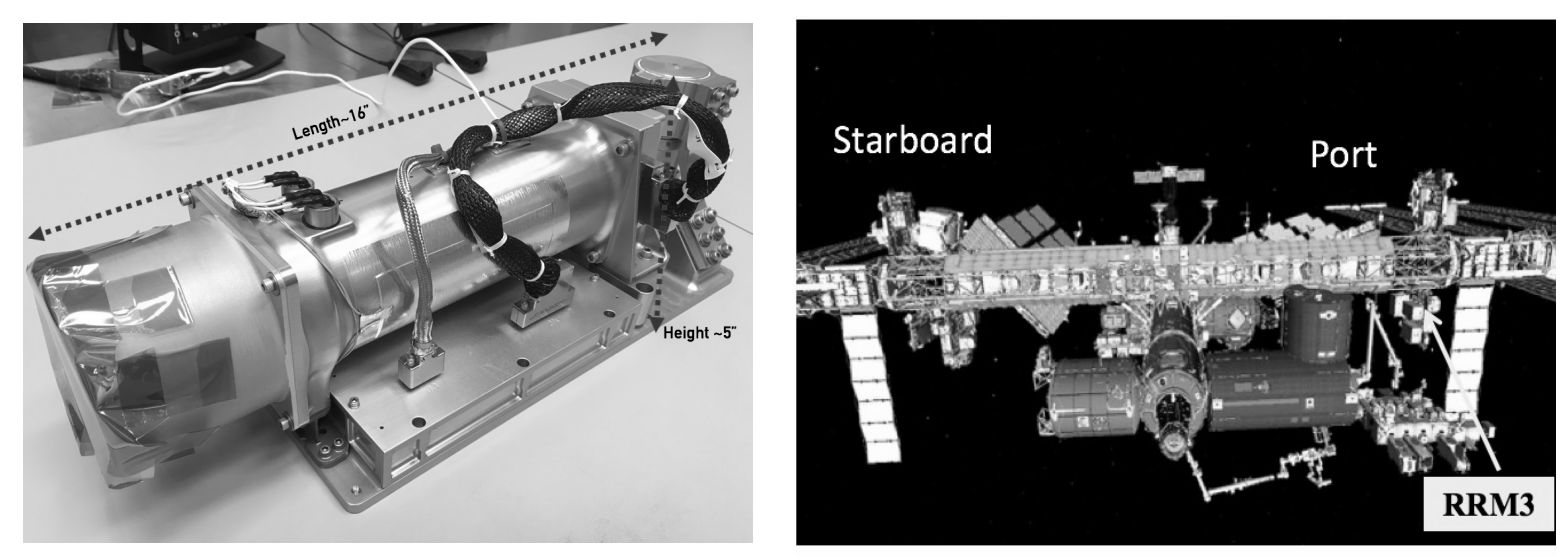

Figure 9. Left; Photograph of the flight CTI instrument with protective lens cover installed. Right; Location of RRM\# (and CTI) on the ISS. 

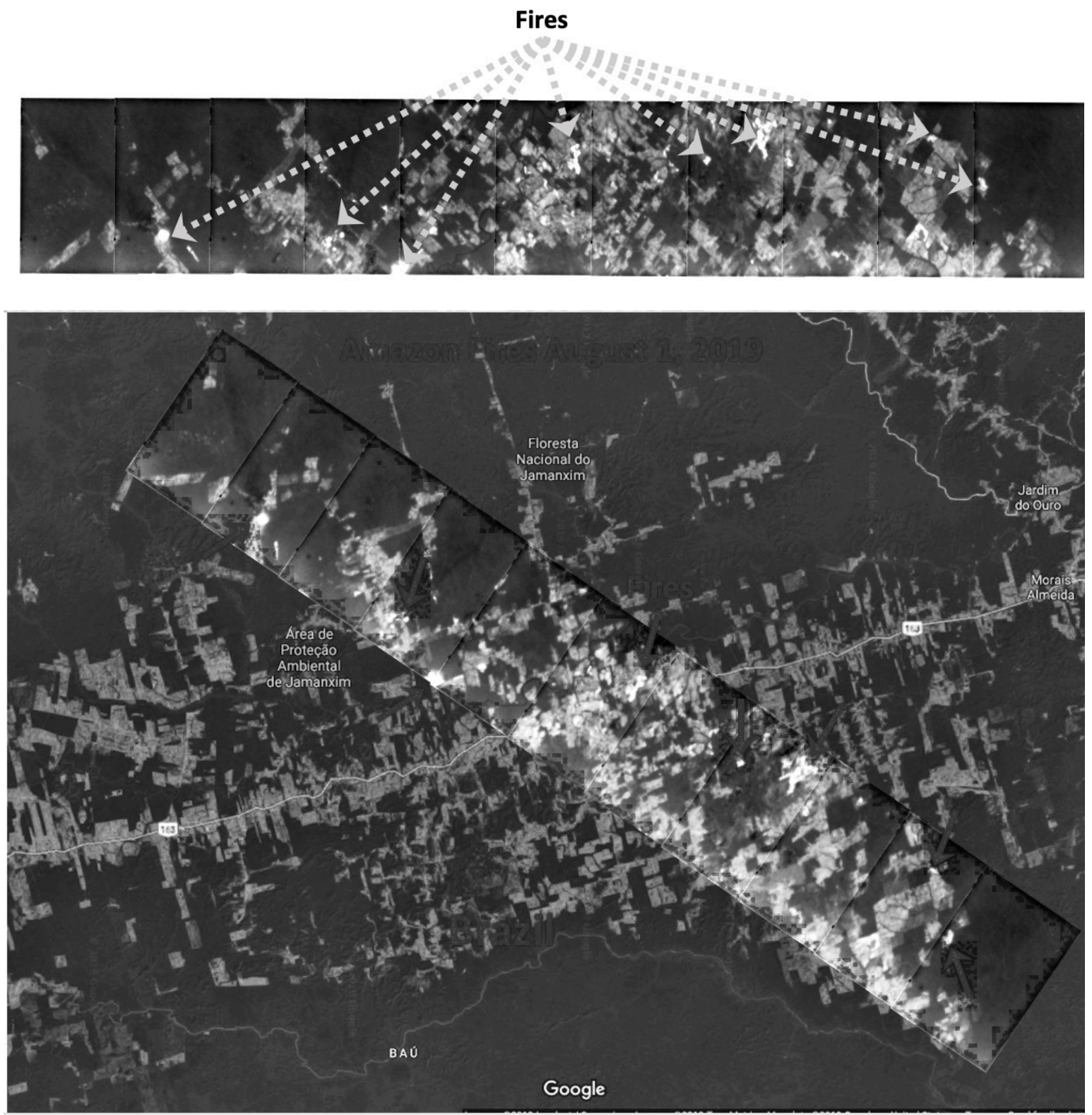

Figure 10. Top image; Sequence of images from CTI band 1 of the fires in the Amazon (over Brazil) taken on August $1^{\text {st }}, 2019$. The bottom composite is the top CTI image sequence layered over the corresponding visible Google Earth image.

\section{Conclusion}

The evolution of quantum well detectors over the last three decades has been dramatic, culminating in their implementation in the NASA legacy Landsat missions. The anticipated advantages of QWIP technology have become a reality; compatibility with existing wafer fabrication methods (commercial and university silicon foundries, for example); high yield, relatively low cost, reliability, uniformity, radiation hardness, stability and ease of scalability, to list some of the more obvious features. Similarly, the development of SLS technology has also been remarkable and much more rapid because of the pathfinding work developing QWIP detectors. In just a few years the SLS technology has evolved from small format arrays to $2 \mathrm{k} \times 2 \mathrm{k}$ hybrids and have performed in a space environment without sacrificing any of the advantages gained by the development of QWIPs with improvements in the sensitivity and operating temperature. We 
continue to develop new instruments implementing SLS arrays in various configurations. Currently, there is an emphasis on multi-band near and far IR imaging on a single focal plane. We are integrating multiple filters directly to the surface of the SLS hybrid to provide precise spectral band selection across the detector. The primary applications for this approach are the detection and analysis of fires in the $4.0 \mu \mathrm{m}$ spectral region and evapotranspiration in the thermal band (10-12 $\mu \mathrm{m})$.

Progress has been substantially enhanced by the collaborative efforts of many groups. Over this span of time we have collaborated with: US Department of Defense, JPL, AT\&T Bell Labs, QmagiQ, L3/Cincinnati Electronics, Lockheed, Rockwell/Teledyne, Raytheon, Northwestern University, George Mason University, Thales Research, NASA/Langley, NASA/Dryden as well as vendors such as Indigo Systems, Intelliepi and IQE.

References

[1] M. Jhabvala, K. Choi, "Evolution of QWIP focal plane development at the NASA/Goddard Space Flight Center, Proc. SPIE 8268, Quantum Sensing and Nanophotonic Devices IX, 82682P (20 January 2012).

[2] K. Forrest and M. Jhabvala, "Gallium Arsenide Quantum Well-based Far Infrared Radiometric Imager". Technology 2001, San Jose, CA, Dec 3-5,1991.

[3] M. Jhabvala, S. Gunapala, D. Reuter, K. K. Choi, S. Bandara, J. Liu, A. La, S. Banks, J. Cho, T. Hwang, S. Tsay, D.

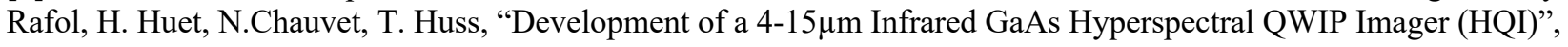
QWIP 2002, Turin, Italy, October, 2002.

[4] M. Sundaram, J. Bundas and A. Reisinger, SBIR Phase I final report; "Handheld Longwave Infrared Camera Based on Highly-Sensitive Quantum Well Infrared Photodetectors", NASA Contract NNX07CA50P, report \# QMV0003, (2007).

[5] M. Jhabvala, D. Reuter, K. Choi, M. Sundaram, C. Jhabvala, A. La, A. Waczynski and J. Bundas", "The QWIP focal plane assembly for NASA's Landsat Data Continuity Mission", SPIE, Orlando, April, 2010.

[6] M. Montanero, "Landsat 8 Thermal Infrared Sensor Focal Plane Array On-Orbit Stability", Internal NASA document, October, 2019.

[7] B. Nguyen, D. Hoffman, Y. Wei, P. Delaunay, A. Hood, and M. Razeghi, "Very high quantum efficiency in type-II InAs/GaSb superlattice photodiode with cutoff of $12 \mu \mathrm{m}$ ", Appl. Phys. Lett., v. 93, issue 23, 2008.

[8] M. Jhabvala, "Development of a Strained-Layer Superlattice IR Detector Camera", NASA/Goddard Internal Research and Development End of Year report, 2013.

[9] "CubeSat Design Specification" California Polytechnic State University, Rev 13, 2009.

[10] M. Sundaram, J. Bundas and A. Reisinger, SBIR Phase I final report; "Dualband mw/lw strained layer superlattice focal plane arrays for satellite-based wildfire detection", NASA Contract NNX14CG38P, report \# QMV0002, (2014).

[11] M. Jhabvala, D. Jennings ${ }^{1}$ C. Tucker, A. La, B. Keer, E. Timmons, R. Stone, T. Flatley, F. Cepollina, S. Babu, A. Lunsford, J. Cassidy, D. Parker ${ }^{3}$ M. Sundaram, J. Bundas, W. Squicciarini, P. Finneran, I. Orlowski, C. Fetter, M. Loose, "The SLS-based Compact Thermal Imager for the International Space Station" Applied Optics, Vol 58, No.20, July 10, 2019.

[12] M. Burks, C. Cork, D. Eckels, E. Hull, N. Madden, W. Miller, J. Goldsten, E. Rhodes, B. Williams, "Thermal Design and Performance of the Gamma-Ray Spectrometer for the MESSENGER Spacecraft", IEEE Symposium Conference Record Nuclear Science, 2004.

[13] T. Flatley, "SpaceCube: A Family of Reconfigurable Hybrid On Board Science Data Processors", presented at the NASA/ESA Conference on Adaptive Hardware and Systems, Nuremberg, Germany, June 2012.

\section{Acknowledgements}

The authors would like to acknowledge the following individuals for their invaluable support to this effort: NASA Headquarters/ESTO,Goddard Space Flight Center,ATT/Bell Labs, Rockwell Science Center/Teledyne, DARPA,LTV, Army Research Lab, Jet Propulsion Lab, Northwestern University, Night Vision Lab, Indigo/FLIR, SE-IR, Intelli-Epi, IQE, L3/Cincinnati Electronics, QmagiQ,Thales, Flightland Data Systems, Thales, Sachi Babu, Jason Bundas, Don Jennings, Christine Jhabvala, George Komar, Anh La, Allen Lunsford, Dennis Reuter, Peter Shu and Kadre Vural. 